\title{
Osteoporosis guideline awareness among Iranian family physicians: results of a knowledge, attitudes, and practices survey
}

\author{
Hamideh Mahdaviazad", Vahid Keshtkar ${ }^{2}$ and Mohammad Jafar Emami ${ }^{3}$ \\ ${ }^{1}$ Assistant Professor, Department of Community and Preventive Medicine, Bone and Joint Diseases Research Center, \\ Shiraz University of Medical Sciences, Shiraz, Iran \\ ${ }^{2}$ Department of Community Medicine, Shiraz University of Medical Sciences, Shiraz, Iran \\ ${ }^{3}$ Professor, Department of Orthopedic Surgery, Bone and Joint Diseases Research Center, Shiraz University of \\ Medical Sciences, Shiraz, Iran
}

\begin{abstract}
Aim: This study investigates the knowledge, attitudes, and practices (KAP) of family physicians in Iran, regarding osteoporosis and their experience with national osteoporosis guideline. Background: Osteoporosis is a relatively preventable, chronic and progressive disease. Family physicians play a crucial role in relieving the burden of care. Methods: This cross-sectional study was addressed at all qualified family physicians who registered at urban family physicians and referral system program. Data collection included demographics, professional experience, and knowledge of guidelines based on a standardized KAP questionnaire. Student's $t$-test was used to measure the associations between KAP scores and demographic, professional experience variables. Findings: The response rate was $72 \%$ (540/750). Based on Bloom's cut off scale, family physicians knowledge and practice scores were in moderate level, and only 14 and $38.5 \%$ of them had good knowledge and practice, respectively. Attitude score was in good level, and $64.1 \%$ of participants had positive attitude. Mean score of knowledge and practice were higher significantly among family physicians that practice in public settings. Family physicians, who completed osteoporosis training courses, had higher attitude score $(P=0.03)$. Only $23.5 \%$ of family physicians were aware of the existence of national osteoporosis guideline. Conclusion: Although most family physicians believed in the importance of preventive measures, however, limited number of them had good knowledge and practice regarding osteoporosis and less than a quarter were aware of national guideline. This is a clear need to disseminate the guideline more effectively, make greater use of efficient training methods.
\end{abstract}

Key words: awareness; family physicians; guideline; knowledge; osteoporosis; practice Received 1 July 2017; revised 11 November 2017; accepted 28 December 2017; first published online 30 January 2018

\section{Introduction}

Osteoporosis is a chronic and progressive disease in which gradual bone loss, microscopic tissue

Correspondence to: Assistant Professor Hamideh Mahdaviazad, Department of Community and Preventive Medicine, Bone and Joint Diseases Research Center, Shiraz University of Medical Sciences, P.O. Box: 7193634154, Shiraz, Iran. Email: mahdavih@sums.ac.ir

(C) Cambridge University Press 2018 destruction, and bone thinning can increase the risk of fracture (World Health Organization, 1994; Wright et al., 2014). Osteoporosis is the most common metabolic bone disorder, which causes hip fracture in the elderly population (De Villiers, 2008; Ioannidis et al., 2009). According to United States Surgeon General's 2004 report, 10 million Americans aged over 50 have osteoporosis that leading to 1.5 million fragility fractures annually (US Department of Health and Human Services, 2004; Curtis et al., 2017). 
In Iran, as in other developing countries, epidemiologic data is lacking (Meybodi et al., 2008) A national 2004 study on the osteoporosis burden, based on the Disability Adjusted Life Years (DALYs) index, showed that the disease was responsible for about 36027 lost years, due to premature mortality and disability (Abolhassani et al., 2004; Meybodi et al., 2008). In 2003, Larijani et al. reported the incidence of hip fracture following a fall as 20.6 and 17.5 per 100000 personyears in males and females, respectively (Larijani et al., 2007). It is clear that the country's growing proportion of older people means that the social and economic burden of osteoporosis will be an increasingly important public health issue (De Villiers, 2008; Romagnoli et al., 2000; Mckercher, 2003; Cheng and Green, 2008).

Family physicians, as a first line of contact with the community, are responsible for application of preventive measures and proper management of chronic diseases like osteoporosis (Fogelman et al., 2016). So their knowledge, attitude, and behavior can play an important role in better management and disease control. A cross-sectional survey among general practitioner in Shiraz showed that only $17 \%$ of them had good knowledge about osteoporosis. The mean of knowledge scores in some important areas including risk factors, diagnosis and treatment were low to intermediate (Hadi, 2005).

At the best of our knowledge, there are no studies to evaluate family physicians awareness or practice regarding osteoporosis in Iran. Current study conducted to evaluate knowledge, attitude, and practice (KAP) of osteoporosis among the family physicians of Shiraz University of Medical Sciences and their experience with national osteoporosis guideline.

\section{Method}

This cross-sectional study was conducted from September to December 2016 in Shiraz, Iran. All qualified urban family physicians who registered in family physician and referral system program affiliated to the Shiraz University of Medical Sciences were included in the study.

The protocol was approved by the University's Ethics Committee. Verbal informed consent was obtained from all participants, after they had been informed of the study's objectives. Complete anonymity and data confidentiality was guaranteed.

Data gathering began in consultation with Vice Chancellor's office; it involved coordination with the family physicians' offices, and obtaining the necessary official permissions. In the second step, supervisors were trained in the distribution and completion of the questionnaires.

Family physicians who did not give consent to participate, or did not attend clinics after three visits were excluded from the study.

The data collection tool was a self-administered, three-part questionnaire developed by the researchers. The first part focused on self-reported demographic and professional practice data; it included the gender (men/women), years of practice as a family physicians, type of practice (public/private), completed osteoporosis training courses (yes/ no). The second part consisted of self-reported questions about guideline experience. It included internet access at work (yes/no), use of electronic resources (yes/no), osteoporosis national guideline availability (yes/no), and guideline study (yes/ no). Third part contained KAP questions, it was based on a review of the relevant literature (Romagnoli et al., 2000), and current national guideline for the diagnosis and management of osteoporosis (National Osteoporosis Research Network, 2014). Knowledge was evaluated from 15 questions that were scored using a threepoint scale $(2=$ correct; $1=$ don't know; $0=$ incorrect $)$. The sum of scores was used as the knowledge score, total score ranged between 0 and 30. Attitude questions (three items) were ranked from 1 to 5 on a five-point Likert scale $(1=$ strongly disagree; $5=$ strongly agree). Participants' score for each question ranged 1-5, and total attitudes score ranged in between 3 and 15 . The six practice questions addressed the referral and follow-up of osteoporotic patients; here, two responses were possible $(0=$ incorrect, $1=$ correct). The sum of scores was used as the practice score, total score ranged between 0 and 6 . We used Bloom's cut off scale to assess level of KAP (Hwaid, 2013). Where mean KAP scores were $80-100 \%$ of total correct responses meant a good knowledge $(\geqslant 24)$, positive attitude $(\geqslant 12)$ and good practice $(\geqslant 5)$. A score of $60-79 \%$ put a scorer in a moderate level and a poor level was for the respondents with a score $<60 \%$ of the total correct responses.

The questionnaire was reviewed by an expert panel composed of three community medicine specialists, and two orthopedic surgeons. 
An epidemiologist checked and improved its validity. Reliability was established in a pretest with 50 participants. Cronbach $\alpha$ coefficient was 0.72 for knowledge questions, 0.71 for attitude questions and 0.70 for practice questions, and it took, on average, 15-20 min to complete.

\section{Statistical analysis}

All data were entered, verified and analyzed using SPSS (Version 16; SPSS Inc., Chicago, IL, USA). Descriptive statistics, including number (\%) and means \pm standard deviation were calculated for responses to demographic, professional experience data. Correlation between KAP scores were assessed by using the Spearman's rank correlation coefficient. Student's $t$-test was used to measure the associations between KAP scores and demographic, professional experience variables. A $P$-value of $<0.05$ was considered statistically significant.

\section{Results}

From total of 750 urban family physicians, 540 of them participated in the survey (response rate: $72 \%$ ). The final analysis concerned 527 completed KAP questionnaires. Among the participants, there were 302 men and 191women. Mean \pm SD year of practice as family physician was $3.72 \pm 1.21$ years. More than two thirds of participants visited patients in private office (367), which is usual practice in Shiraz. Only 161 of family physicians (30.6\%) completed osteoporosis training courses.

Table 1 summarize the frequency of participants' responses to KAP questions. The overall mean scores $\pm \mathrm{SD}$ for all participants were $17.9 \pm 4.8$ for knowledge, $12.0 \pm 1.8$ for attitudes and $3.9 \pm 1.4$ for practices. Based on Bloom's cut off scale, family physicians knowledge and practice scores were in moderate level, and only 14 and $38.5 \%$ of them had good knowledge and practice, respectively. Attitude score was in good level, and $64.1 \%$ of participants had positive attitude.

Based on Pearson correlation coefficients, there were significant positive linear correlations between knowledge and attitude scores (correlation coefficient: $0.15, P<0.001)$, knowledge and practice scores (correlation coefficient: 0.28; $P<0.001$ ), attitude and practice scores (correlation coefficient: $0.20 ; P<0.001)$.
Table 2 presents the results of association between KAP scores and demographic and professional variables. Mean KAP scores among female family physicians were higher than those who were male; however, these differences were not statistically significant. Mean score of knowledge was $18.8 \pm 4.6$ among family physicians who practice in public setting and $17.5 \pm 4.7$ among those who practice in private setting $(P=0.01)$. Practice scores were $4.2 \pm 1.3$ and $3.8 \pm 1.4$ among family physicians in public and private setting, respectively, and this difference was statistically significant $(P=0.01)$. Family physicians, who completed osteoporosis training courses had higher attitude score than those who not completed courses $(12.3 \pm 1.6$ versus $12.3 \pm 1.6 ; P=0.03)$.

Figure 1 shows family physicians experience with national guideline. Only $23.5 \%$ (124) of family physicians were aware of the existence of national osteoporosis guideline. Of these, $17.6 \%$ (93) had studied them and used them in their practice. Despite the high availability of the internet $(92.2 \%)$, the use of electronic resources was only $37.2 \%$ among family physicians.

\section{Discussion}

Osteoporosis is relatively preventable disease, which is major contributor to mortality and morbidity in the elderly population (Saeedi et al., 2014). The primary healthcare system and evidence-based medicine both have a clear role in decreasing the burden of this disease (Chenot et al., 2007). In this context, the Iranian Osteoporosis Research Network was established to coordinate national osteoporosis research programs (Meybodi et al., 2008). Furthermore, in 2007, the Endocrinology and Metabolism Research Center developed national clinical guidelines for evidence-based practice targeted at family physicians (National Osteoporosis Research Network, 2014).

Our results revealed that mean score of knowledge among urban family physicians regarding osteoporosis was in moderate level, and only $14 \%$ of them had good level of knowledge. The result for knowledge was consistent with results reported by Hadi and Shiralli (2005) and Chenot et al. (2007). The results of both studies found that primary care doctors have average knowledge regarding osteoporosis. 
Table 1 Frequency of participants' responses to knowledge, attitude and practice questions

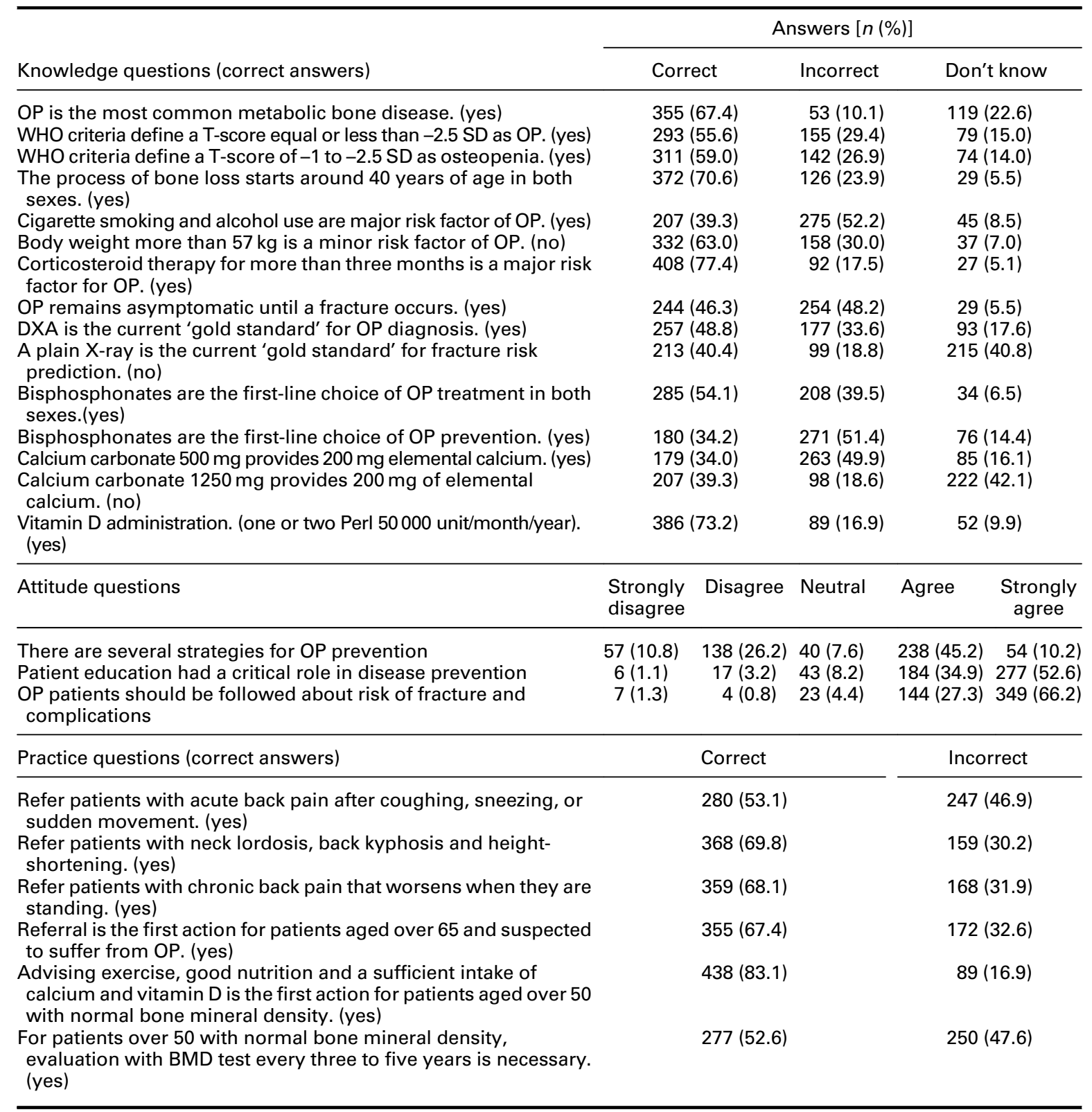

$\mathrm{OP}=$ osteoporosis; $\mathrm{DXA}=$ dual $\mathrm{X}$-ray absorptiometry; $\mathrm{SD}=$ standard deviation; $\mathrm{BMD}=$ bone mineral density.

Regarding attitude, approximately two third of family physicians had positive attitude regarding existence of several strategies for prevention, education, and follow-up (or risk assessments) of osteoporotic patients. These results are consistent with earlier literature which reports that most physicians are optimistic about the preventable nature of osteoporosis, have a positive attitude to the critical role of primary healthcare, and their duty in terms of the diagnosis and follow-up of 
Table 2 Mean of knowledge, attitude and practice scores toward osteoporosis by demographic and professional data

\begin{tabular}{|c|c|c|c|c|c|c|}
\hline \multirow[t]{2}{*}{ Variables } & \multicolumn{2}{|c|}{ Knowledge score } & \multicolumn{2}{|c|}{ Attitude score } & \multicolumn{2}{|c|}{ Practice score } \\
\hline & Mean \pm SD & $P$-value & Mean \pm SD & $P$-value & Mean \pm SD & $P$-value \\
\hline \multicolumn{7}{|l|}{ Gender } \\
\hline Men & $17.6 \pm 4.8$ & 0.29 & $12.0 \pm 1.8$ & 0.27 & $3.9 \pm 1.4$ & 0.72 \\
\hline & \multicolumn{5}{|c|}{ Years of practice } & \\
\hline $\begin{array}{l}\leqslant 3 \text { years } \\
>4 \text { years }\end{array}$ & $\begin{array}{l}18.4 \pm 4.8 \\
17.6 \pm 4.8\end{array}$ & 0.11 & $\begin{array}{l}12.0 \pm 1.8 \\
12.1 \pm 1.8\end{array}$ & 0.75 & $\begin{array}{l}3.9 \pm 1.5 \\
3.9 \pm 1.3\end{array}$ & 0.89 \\
\hline \multicolumn{7}{|c|}{ Type of practice } \\
\hline $\begin{array}{l}\text { Public } \\
\text { Private }\end{array}$ & $\begin{array}{l}18.8 \pm 4.6 \\
17.5 \pm 4.7\end{array}$ & 0.01 & $\begin{array}{l}12.0 \pm 1.9 \\
12.1 \pm 1.8\end{array}$ & 0.71 & $\begin{array}{l}4.2 \pm 1.3 \\
3.8 \pm 1.4\end{array}$ & 0.01 \\
\hline \multicolumn{7}{|c|}{ Osteoporosis training course } \\
\hline $\begin{array}{l}\text { Yes } \\
\text { No }\end{array}$ & $\begin{array}{l}18.2 \pm 4.4 \\
17.7 \pm 4.9\end{array}$ & 0.31 & $\begin{array}{l}12.3 \pm 1.6 \\
11.9 \pm 1.9\end{array}$ & 0.03 & $\begin{array}{l}3.8 \pm 1.4 \\
3.9 \pm 1.4\end{array}$ & 0.25 \\
\hline
\end{tabular}

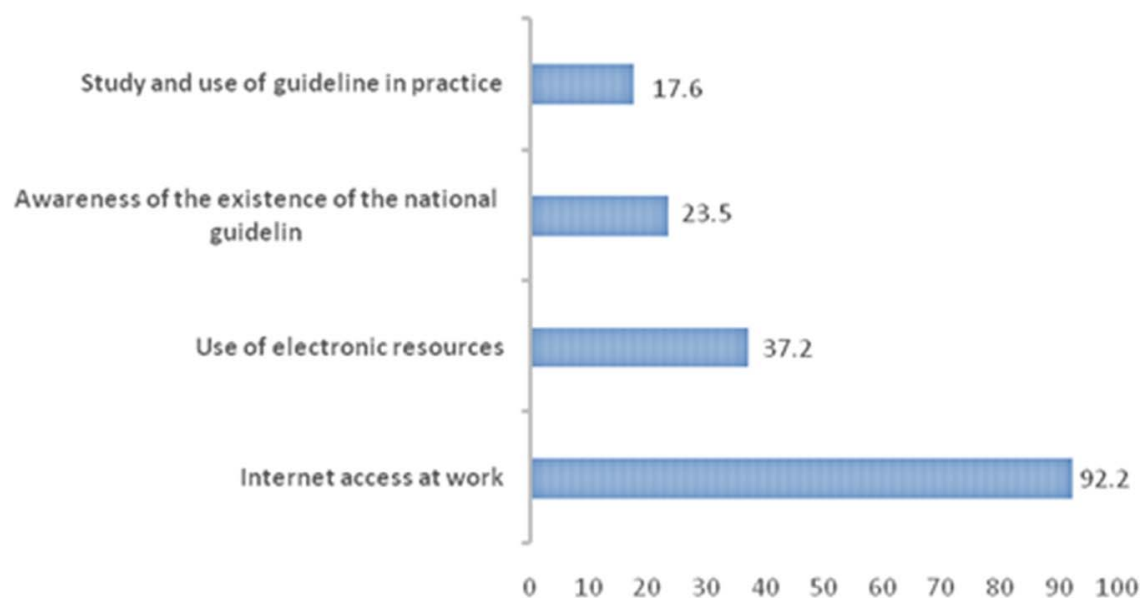

Figure 1 Physician's experience of osteoporosis guideline

osteoporotic patients (Hadi, 2005; Beshyah et al., 2013; Saeedi et al., 2014).

In terms of practice, mean score of practice was in moderate level and $38.5 \%$ of participants had good practice in osteoporotic patients referring. Jaglal et al. explored Canadian family physicians' experience of osteoporosis using focus groups. This study noted that family physicians were confused about the time to start treatment, the safety of drugs, and adherence to drug regimens (Jaglal et al., 2003). Beshyah et al. (2013) reported that United Arab Emirates physicians use of outdated approach to management of osteoporosis. However, practice scores were not reported in these studies, or other similar reports. This, together with differences in methodology and the limited number of studies makes it difficult to draw comparisons.

The association between KAP scores and type of practice and osteoporosis training course were statistically significant. Family physicians that practice in public setting had higher mean scores in knowledge and practice $(P=0.01)$. This higher score could be related to more restricted supervisions in public setting of our healthcare system. Participants who completed osteoporosis training courses had only higher mean score in attitude $(P=0.03)$. This finding indicates that knowledge 
and attitude of family physicians not translated into practice well. So, use of efficient training methods and evidence-based guidelines stressed.

Regarding national osteoporosis guideline, surprisingly $76.5 \%$ of family physicians stated that they were unaware of the existence of clinical guideline. Less than a third use national guideline or draw upon other electronic resources. These results are in line with the level of awareness of guidelines among physicians on Saudi Arabia and the United Arab Emirates (16 and 25\%) (Beshyah et al., 2013; Saeedi et al., 2014). This can be compared with research from Germany and Canada, which suggests that guidelines are used by nearly $50 \%$ of physicians. In the United Kingdom, only a quarter of general practitioners rarely, or never, use osteoporosis guidelines (Taylor et al., 2001; Jaglal et al., 2003; Chenot et al., 2007). The lack of awareness or use of guidelines in our setting may be explained by how they are advertised or disseminated, and problems related to their content, which is not designed to be user friendly.

\section{Strengths and limitations}

To the best of our knowledge, this is the first study of osteoporosis KAP among family physicians in Iran. As all urban family physicians in the healthcare system were included, the results provide valid input for policymakers. Response rates were excellent, as the project was presented as an educational needs assessment. This meant that participants did not feel that they were being examined, or their competence was being questioned. The main limitation was the small number of publications in this area, which made comparisons difficult.

\section{Conclusion}

We concluded that limited number of urban family physicians had good knowledge and practice regarding osteoporosis. This was observed despite that we know adequate and effective management of osteoporosis is necessary considering to burden of problem in our country. The results also highlight a lack of awareness and use of national guidelines. These may reflect deficits in the prevention and control of osteoporosis. Therefore, it is important to find a more effective way to disseminate national osteoporosis guidelines and extend the use of efficient training methods such as problem-based learning, self-directed learning, and interactive workshops, which may contribute to decreasing the burden of the disease. A further recommendation is greater access to the internet and encouraging physicians to use electronic resources. Further research will be required to evaluate the effectiveness of these new interventions.

\section{Acknowledgments}

The authors very much appreciate the cooperation offered by the Health Vice Chancellor and Office of Family Physicians, who provided them with essential logistic support. Without their help, the authors could not have performed this study. Finally, they thank Elaine Seery (AuthorAID in the Eastern Mediterranean) for improving the use of English in the manuscript.

\section{Financial Support}

The project was financed by Vice Chancellor for Research of the Shiraz University of Medical Science for their financial support (Grant No. 95-01-56-12121).

\section{Conflicts of Interest}

The authors declare that they have no conflict of interest.

\section{Ethical Standards}

The protocol was approved by the University's Ethics Committee. Verbal informed consent was obtained from all participants, after they had been informed of the study's objectives. Complete anonymity and data confidentiality was guaranteed.

\section{References}

Abolhassani, F., Mohammadi, M. and Soltani, A. 2004: Burden of osteoporosis in Iran. Iranian Journal of Public Health, 18-28.

Beshyah, S.A., Mehri, W.A. and Khalil, A.B. 2013: Osteoporosis and its management: knowledge, attitudes and practices of physicians in United Arab Emirates. Ibnosina Journal of Medical and Biomedical Sciences 5, 270-79.

Cheng, N. and Green, M.E. 2008: Osteoporosis screening for men: are family physicians following the guidelines? Canadian Family Physician 54, 1140-141. 
Chenot, R., Scheidt-Nave, C., Gabler, S., Kochen, M. and Himmel, W. 2007: German primary care doctors' awareness of osteoporosis and knowledge of national guidelines. Experimental and Clinical Endocrinology \& Diabetes 115, 584-89.

Curtis, E.M., Moon, R.J., Harvey, N.C. and Cooper, C. 2017: The impact of fragility fracture and approaches to osteoporosis risk assessment worldwide. Bone 104, 29-38.

De Villiers, T.J. 2008: Preventing osteoporosis in postmenopausal women: treatment approaches for family physicians. South African Family Practice 50, 41-47.

Fogelman, Y., Goldshtein, I., Segal, E. and Ish-Shalom, S. 2016: Managing osteoporosis: a survey of knowledge, attitudes and practices among primary care physicians in Israel. PLoS One 11, e0160661.

Hadi, N. and Shiralli, R. 2005: Level of knowledge and attitude toward osteoporosis among general physicians in Shiraz 2003. Hormozgan Medical Journal 4, 373-78. [In Persian].

Hwaid, A.H. 2013: Knowledge and awareness of papillomavirus and cervical cancer among college students and health care workers women in Diyala, Iraq. American Journal of Public Health Research 1, 221-25.

Ioannidis, G., Papaioannou, A., Thabane, L., Gafni, A., Hodsman, A., Kvern, B., Walsh, A., Jiwa, F. and Adachi, J.D. 2009: Family physicians' personal and practice characteristics that are associated with improved utilization of bone mineral density testing and osteoporosis medication prescribing. Population Health Management 12, 131-38.

Jaglal, S.B., Carroll, J., Hawker, G., Mcisaac, W.J., Jaakkimainen, L., Cadarette, S.M., Cameron, C. and Davis, D. 2003: How are family physicians managing osteoporosis? Qualitative study of their experiences and educational needs. Canadian Family Physician 49, 462-68.

Larijani, B., Resch, H., Bonjour, J., Meybodi, H.A. and Tehrani, M.M. 2007: Osteoporosis in Iran, overview and management. Iranian Journal of Public Health 1-13.

Mckercher, H. 2003: Family physicians and osteoporosis. Meeting the challenge. Canadian Family Physician 49, 405.
Meybodi, H.A., Heshmat, R., Maasoumi, Z., Soltani, A., Hossein-Nezhad, A., Keshtkar, A., Bahrami, A., Rajabian, R., Nabipour, I. and Omrani, G. 2008: Iranian osteoporosis research network: background, mission and its role in osteoporosis management. Iranian Journal of Public Health 37, 1-6.

National Osteoporosis Research Network. 2014: Osteoporos clinical guideline. Retrieved 23 January 2018 from http://vch. iums.ac.ir/uploadesteoproz.pdf. [in persian]

Romagnoli, E., Colangeli, I. and Minisola, S. 2000: Awareness, attitudes and opinions on osteoporosis of primary care physicians working in the metropolitan area of Rome: a brief report. Aging Clinical and Experimental Research 12, 240-44.

Saeedi, M.Y., Al-Amri, F., Mohamed, A. and Ibrahim, A.K. 2014: Knowledge, attitude and practice towards osteoporosis among primary health care physicians in Riyadh, Saudi Arabia. Science Journal of Public Health 2, 624-30.

Taylor, J., Sterkel, B., Utley, M., Shipley, M., Newman, S., Horton, M. and Fitz-Clarence, H. 2001: Opinions and experiences in general practice on osteoporosis prevention, diagnosis and management. Osteoporosis International 12, 844-48.

US Department of Health and Human Services. 2004: Bone health and osteoporosis: a report of the Surgeon General. Rockville, MD: US Department of Health and Human Services, Office of the Surgeon General, $87 \mathrm{pp}$.

World Health Organization (WHO). 1994: Assessment of fracture risk and its application to screening for postmenopausal osteoporosis: report of a WHO study group, meeting held in Rome, 22 to 25 June 1992.

Wright, N.C., Looker, A.C., Saag, K.G., Curtis, J.R., Delzell, E.S., Randall, S. and Dawson-Hughes, B. 2014: The recent prevalence of osteoporosis and low bone mass in the United States based on bone mineral density at the femoral neck or lumbar spine. Journal of Bone and Mineral Research 29, 2520-526. 